operational definition and Core Outcome Set for wellbeing in doctors is needed to meaningfully progress the work in this field. Method. The Centre for Workforce Wellbeing (C4WW), a collaboration between the University of Southampton and Health Education England, was created to support research into the nature, assessment and enhancement of wellbeing in physicians. A Systematic Review of wellbeing measures used in doctors and the robustness of those measures, along with surveys of $250 \mathrm{UK}$ doctors of all grades and specialities and patient and public involvement is informing what a core outcome set could be. A Delphi Study among 37 UK experts has been initiated to establish the consensus Core Outcome Set.

Result. Publication of research into doctors' wellbeing is growing internationally. In the UK alone data are being captured by multiple national organisations including: the Care Quality Commission, General Medical Council, British Medical Association and the Royal Colleges. Health and Social Care Organisations are, therefore, keen to "do something" and are spending money on wellbeing interventions with little, or no, evidence base and a lack of appropriate, comparable evaluation. A Core Outcome Set for measuring wellbeing in doctors is ethically required to reduce waste, to replace burnout measures and to refine wellbeing interventions.

Conclusion. Wellbeing measures that actually measure wellbeing, and not burnout, which are validated, reliable and practical, are needed to inform local organisational, national government and international research policy. An absence of burnout does not equate to wellbeing. The focus of measurement needs to shift to capture in what contexts we thrive, not just survive. If everyone used the same Core Outcome Set to measure mental wellbeing, direct comparisons could be made, and money invested, in creating infrastructure, processes and cultures that really work.

Health Education England funded PhD.

\section{What is mental wellbeing?}

\section{David Baldwin ${ }^{1 \star}$, Julia Sinclair ${ }^{1}$ and Gemma Simons ${ }^{2}$}

${ }^{1}$ Professor of Psychiatry and Head of Mental Health Group, University of Southampton Faculty of Medicine Honorary Professor of Psychiatry, University of Cape Town, South Africa Visiting Professor, Shandong Mental Health Centre, China and ${ }^{2}$ Centre for Workforce Wellbeing, University of southampton

${ }^{*}$ Corresponding author.

\section{doi: 10.1192/bjo.2021.631}

Aims. To explore the theory of wellbeing and to propose an operational definition for wellbeing in doctors.

Hypothesis: An operational definition for wellbeing in doctors is needed in order for it to be measured and interventions to improve it developed.

Background. There is no internationally recognised definition for wellbeing and yet wellbeing is an increasingly fashionable topic of research and development, including in doctors. This is because wellbeing can be described using either hedonist, or eudonist philosophy and there is a lack of conceptual clarity about what wellbeing is, and how it works. Research into the measurement of mental wellbeing has been dominated by individualist societies, with the inherent bias towards measuring self-centred components and not the other-orientated components that might be valued more in collectivist societies and by doctors.

Method. The Centre for Workforce Wellbeing (C4WW), a collaboration between the University of Southampton and Health Education England, was created to support research into the nature, assessment and enhancement of wellbeing in physicians. A literature review of the philosophy, definition and measurement of wellbeing was undertaken with a focus on mental wellbeing at work and specifically in doctors.

Result. A concept map of the relationship between wellbeing terms has been created and was used to understand and classify where mental wellbeing itself was being defined and measured in studies, as opposed to a component of wellbeing, or determinant of wellbeing. Thematic analysis was used to develop an operational definition of wellbeing for doctors.

Conclusion. Measurement of wellbeing and interventions for wellbeing cannot be developed if you cannot clearly define what wellbeing is. An operational definition of mental wellbeing in doctors is ethically required to prevent research waste and to allow us to identify and recreate when doctors thrive, not just survive.

Health Education England funded $\mathrm{PhD}$.

\section{A state hospital survey of movement disorders including intention tremor}

\author{
Nigel Bark ${ }^{1 *}$, Sung-Ai Kim² and George Eapen ${ }^{3}$ \\ ${ }^{1}$ Albert Einstein College of Medicine, Bronx Psychiatric Center; \\ ${ }^{2}$ Bronx Psychiatric Center (Retired) and ${ }^{3}$ Rainbow Babies and \\ Childrens Hospital \\ ${ }^{*}$ Corresponding author.
}

doi: 10.1192/bjo.2021.633

Aims. In a survey of movement disorders in patients in a State Hospital the finger-nose test was included because of increasing interest in the cerebellum in schizophrenia. It was expected that this would reflect the pathobiology of schizophrenia and be unrelated to the type of medication.

Background. Abnormalities of movement and involuntary movements have gone from being considered part of schizophrenia to side-effects of medication to now demonstrably present in those who have never taken anti-psychotic medication. Soft neurological signs (SNS) are increased in schizophrenia, unrelated to medication, considered not to indicate brain localization, yet often include the finger-nose test which localizes to the cerebellum.

Method. All available patients in a State Hospital were examined for movement disorders. They were rated on the following scales: Abnormal Involuntary Movement Scale (AIMS) for Tardive Dyskinesia (TD), Simpson-Angus Neurological Rating Scale for Parkinsonism (SANRS), Barnes Akathisia Scale (BAS), a Dystonia scale and the finger-nose test.

Result. 250 patients were included, 174 were examined or observed for movement disorder: 120 had no missing data, 54 refused part of the exam. Their mean age was $47,62 \%$ male, $53 \%$ black, 26\% Hispanic, 17\% white.

Medication: First Generation Antipsychotic (FGA) 35 (mean CPZ equivalent dose:1177mg), Second Generation Antipsychotic (SGA) 159 (734mg), both FGA and SGA 56 (1907mg), no antipsychotic 3; anticholinergic or amantidine: FGA 57\%, SGA $16 \%$, both FGA and SGA: $50 \%$.

Tardive Dyskinesia: all 23\%, FGA 36\%, SGA 25\%, both 7\%

Parkinsonism: all 38\%, FGA 43\%, SGA 33\%, both 34\%

Akathisia: all 3\%, FGA 0\%, SGA 4\%, both 3\%

Pseudo-akathisia: FGA $11 \%$, SGA $4 \%$, both $13 \%$

Dystonia: all $10 \%$, FGA 13\%, SGA $11 \%$, both $8 \%$

Intention Tremor: all 16\%, FGA 0\%, SGA $21 \%$, both $16 \%$

Half of those with Intention Tremor had Parkinsonism, a third had TD and a half were on anti-Parkinson medication.

None of these differences were statistically significant at $\mathrm{p}=0.05$ though intention tremor did show a trend $(p=0.08)$. The difference between FGA and SGA only became significant when all movement disorders were added together with those on anticholinergics with no movement disorder. 
When compared with rates in similar State Hospitals in the 1970s tardive dyskinesia was now half the rate and Parkinsonism about the same.

Conclusion. Overall rates of movement disorder are not very different between FGA and SGA. The surprise was that intention tremor only occurred with SGAs. Why?

\section{The effect of ketamine on psychopathology and implications for understanding schizophrenia and its therapeutic use: a meta-analysis}

Katherine Beck ${ }^{1 \star}$, Guy Hindley ${ }^{1}$, Faith Borgan ${ }^{1}$, Cedric Ginestet ${ }^{1}$, Robert McCutcheon ${ }^{1}$, Stefan Brugger ${ }^{1}$, Naomi Driesen ${ }^{2}$, Mohini Ranganathan ${ }^{2}$, Deepak D'Souza ${ }^{2}$, Matthew Taylor ${ }^{3}$, John Krystal ${ }^{2}$ and Oliver Howes ${ }^{1}$

${ }^{1}$ IoPPN King's College London; ${ }^{2}$ Yale University School, Dept. of Psychiatry and National Centre for PTSD, VA Connecticut and

${ }^{3}$ University Department of Psychiatry, Warneford Hospital ${ }^{\star}$ Corresponding author.

doi: 10.1192/bjo.2021.634

Aims. To conduct a meta-analysis of the effect of ketamine on psychopathology in healthy volunteers and patients with schizophrenia, and the experimental factors affecting this.

Background. Ketamine is increasingly used to treat depression and other psychiatric disorders but can induce schizophrenia-like symptoms. Despite this, the consistency and magnitude of symptoms induced by ketamine, or what factors influence the effects of ketamine on these remain unknown.

Method. MEDLINE, EMBASE and PsychINFO databases were searched for within-subject placebo controlled studies reporting symptoms using the Brief Psychiatric Rating Scale (BPRS) or Positive and Negative Syndrome Scale (PANSS) in response to an acute ketamine challenge in healthy participants or people with schizophrenia. Two independent investigators extracted study-level data for a random-effects meta-analysis. Total, positive and negative BPRS and PANSS scores were extracted. Sub-group analyses were conducted examining the effect of: blinding status, ketamine preparation, infusion method and time between ketamine and placebo condition. Standardized mean change scores were used as effect sizes for individual studies. Standardized mean changes between ketamine and placebo for total, positive and negative BPRS and PANSS were calculated.

Result. Of 7819 citations retrieved, 36 studies involving healthy participants were included. The overall sample included 725 healthy volunteers exposed to both the ketamine and placebo condition. Ketamine induced a significant increase in transient psychopathology in healthy participants, for total (Standardized mean change $(\mathrm{SMC})=1.50(95 \% \mathrm{CI}=1.23$ to 1.77$), \mathrm{p}<0.0001)$, positive $(\mathrm{SMC}=$ $1.55(95 \% \mathrm{CI}=1.29$ to 1.81$), \mathrm{p}<0.0001)$ and negative $(\mathrm{SMC}=1.16$, (95\% $\mathrm{CI}=0.96$ to 1.35$), \mathrm{p}<0.0001$ ) symptom ratings, relative to the placebo condition. This effect was significantly greater for positive symptoms than negative symptoms $(p=0.004)$. Bolus followed by constant infusion increased ketamine's effect on positive symptoms relative to infusion alone $(\mathrm{p}=0.006)$. Single-day study design increased ketamine's effect on total symptoms $(p=0.007)$, but age and gender did not moderate effects. There were insufficient studies for meta-analysis of studies in schizophrenia. Of these studies, two found a significant increase in symptoms with ketamine administration in total and positive symptoms. Only one study found an increase in negative symptom severity with ketamine.

Conclusion. These findings show that acute ketamine administration induces schizophrenia-like symptomatology with large effect sizes but there is a greater increase in positive than negative symptoms, and when a bolus is used. These findings suggest bolus doses should be avoided in its therapeutic use to minimize the risk of inducing transient positive psychotic symptoms.

\section{Evaluation of self-esteem in adolescents of secondary school level}

Farhana Begum ${ }^{1 \star}$, Usama Zubair ${ }^{2}$, Iqbal Afridi ${ }^{3}$, Fatima Toufique ${ }^{4}$, Jawed Dars ${ }^{3}$ and Chunni Lal ${ }^{3}$

${ }^{1}$ Patel Hospital; ${ }^{2}$ Conolly Hospital; ${ }^{3}$ Jinnah Postgraduate Medical Centre and ${ }^{4}$ Sindh Rangers Hospital

${ }^{\star}$ Corresponding author.

doi: 10.1192/bjo.2021.635

Aims. To assess the frequency of low self-esteem among adolescents of secondary school level in private schools of Karachi Method. It was a cross-sectional descriptive study done in ten private schools of Karachi for a period of 6 months. The proposal of the study was accepted by ethical committee of Jinnah Postgraduate Medical Centre, Karachi (called Institutional Review Board or IRB).The subjects fulfilling inclusion criteria were enrolled after obtaining informed consent by their parents. A semi-structured Performa was used to assess students' particulars and included Rosenberg Self-esteem Questionnaire as a part of Performa. The data were analysed using SPSS version 17.0. Frequencies \& percentages were generated for the level of self-esteem.Stratified analysis was done with a $\mathrm{p}$ value $<0.05$ taken as significant.

Result. Out of the 246 students, $39.8 \%$ were of 14 yrs of age, $36.2 \%$ were 15 years of age, while only $24 \%$ of adolescents were 16 years of age. Majority $(64.2 \%)$ of the students were males while females were $35.8 \% .70 .3 \%$ of the students had normal level of self-esteem, whereas $28.9 \%$ of students had low self-esteem and only $0.8 \%$ of students had high self-esteem. Relationship of all the variables was found to be non-significant except that of educational level ( $p$-value 0.047). Conclusion. Self-esteem was found to be normal in most of the adolescents of secondary school level but still more than $1 / 4$ th of the study participants had low self-esteem which if assessed and addressed early may save the individuals from mental health issues as well as problems at work and home life.Having a better understanding of self-esteem, can help us to identify the adolescents who have low self-esteem and are predisposed to develop mental health difficulties in future.It can lead to not only early intervention and reducing the burden of disease but also help in developing programs to help improve self-esteem in adolescents,hence increasing their overall motivation and productivity.

\section{Mental health, physical impairment and violence} among FSWS in North Karnataka, South India: a story of intersecting vulnerabilities

Alicja Beksinska ${ }^{1 \star}$, Tara S Beattie ${ }^{1}$, Lucy Platt ${ }^{2}$, Parinita Bhattacharjee ${ }^{3}$, Ravi Prakash ${ }^{3}$, Satyanarayana Ramanaik ${ }^{4}$, Kavitha Dibbadahalli ${ }^{3}$, Martine Collumbien ${ }^{1}$, Mitzy Gafos ${ }^{1}$, Calum Davey ${ }^{2}$, Charlotte Watts ${ }^{1}$, Shajy Isac ${ }^{4}$ and Rachel Jewkes ${ }^{5}$

${ }^{1}$ Department of Global Health and Development, London School of Hygiene and Tropical Medicine; ${ }^{2}$ Department of Public Health, Environments and Society, London School of Hygiene and Tropical Medicine; ${ }^{3}$ Karnataka Health Promotion Trust; ${ }^{4}$ Karnataka Health Promotion Trust, University of Manitoba, Department of Community Health Sciences and ${ }^{5}$ Gender and Health Division, South African Medical Research Council

${ }^{\star}$ Corresponding author.

doi: 10.1192/bjo.2021.636 